JEEP 2009, 00004 (2009)

DOI:10.1051/jeep/200900004

(C) Owned by the authors, published by EDP Sciences, 2009

\title{
Analyse thermodynamique des terres rares et leurs alliages $\operatorname{REIn}_{3}$ ${ }^{a}$ V.P. Vassiliev, ${ }^{b}$ A.F. Taldrik, ${ }^{\mathrm{C}}$ B. Legendre
}

a Laboratoire de Thermodynamique Chimique, Faculté de Chimie, Université Lomonossov, Moscou 119992, Russie.E-

c_ mail: vas@td.chem.msu.ru b Institut des Alliages Solides, Moscou.

Laboratoire de Chimie Physique Minérale et Bioinorganique, EA 401, Faculté de Pharmacie, Université de Paris XI, 5 rue J.-B. Clément, F-92296, Châtenay-Malabry, France.

\begin{abstract}
L'analyse thermodynamique des terres rares et leurs alliages avec l'indium est réalisée à l'aide des méthodes corrélatives pour les phases isostructurales RE et REIn ${ }_{3}$. La méthode corrélative proposée de l'estimation des propriétés physico-chimique convient pour toutes les phases isostructurales RE avec d'autres éléments.

Les valeurs des grandeurs thermodynamiques calculées sont recommandées pour les " hand books » destinés aux métallurgistes.

Keywords : Thermodynamic properties, RE pure, alloys $R E \ln _{3}$.
\end{abstract}

\section{Introduction}

Une étude plus approfondie de la loi périodique consiste à interpréter et généraliser la base des données sur les structures et les propriétés des phases simples et complexes. Dans ce travail nous avons étudié des exemples de corrélations entre des analyses thermodynamiques et physico-chimique. Certaines propriétés thermodynamiques ont été étudiées par l'un des auteurs [1].

La meilleure corrélation du type 'propriété thermodynamiques -propriété physiques' est donnée par les relations entre l'enthalpie de formation, l'enthalpie libre et l'entropie standard, les enthalpies de transformations de phases, largueur de la zone interdite etc. d'une part et, d'autre part des propriétés comme le numéro atomique de l'élément, la masse moléculaire, la plus courte distance entre les atomes $A$ et $B$ dans la maille cristalline, la température de fusion du composé défini $A_{n} B_{m}$. Nous pouvons considérer le premier groupe des propriétés comme une fonction et celles du deuxième comme un argument. Une étude expérimentale du premier groupe demande beaucoup de moyens matériels, tandis que les propriétés du deuxième groupe sont bien connues ou assez standardisées.

Donc, les méthodes corrélatives et celles de l'évaluation comparatives sont nécessaire lors de l'analyse des propriétés thermodynamiques et physicochimiques des phases isostructurales, y compris les méthodes d'optimisation des diagrammes de phases. Une telle analyse permet aussi la planification des expériences et elle peut attirer l'attention sur les désaccords entre différentes données. Par exemple, l'utilisation de données d'optimisation peu fiables pour les systèmes binaires $\mathrm{Ga}-\mathrm{As}, \mathrm{Ga}-\mathrm{P}$ et $\mathrm{Ga}-\mathrm{Sb}$ [2] conduit à des descriptions incorrectes des systèmes ternaires Ga-In-P, Ga-As-P et Ga-P-Sb [3].

L'analyse critique des données expérimentales et de celles issues du calcul concernant les fonctions thermodynamiques des phases intermédiaires a permis d'établir la conformité pour des phases de même type pour les systèmes $A^{\prime \prime \prime}-B^{V}, A^{I I}-B^{V l}, A^{I I I}-B^{V I}, R E-M e(M e=$ In, $\mathrm{Pb}, \mathrm{Sb}$ et d'autres éléments), le coefficient de corrélation est de 0.99 selon les critères cristallochimiques. Cela permet le d'obtenir des données thermodynamiques avec un haut degré de fiabilité et d'évaluer les propriétés physico-chimiques des phases qui n'ont pas été mesurées.

Autrement dit, l'état actuel de la science nécessite de réaliser une optimisation de tout un ensemble de données pour parvenir à une cohérence des systèmes étudiés, dans le cadre d'un model thermodynamique unique.

Nous allons aborder quelques exemples qui illustrent la conformité avec la loi périodique.

\section{Terres rares et leurs alliages}

Une étude parfaite des propriétés physicochimiques des terres rares et de leurs alliages avec d'autres éléments est rendue difficile pour les deux raisons suivantes:

1. une grande activité chimique des terres rares (RE) et de leurs alliages (réaction chimique avec l'air et l'humide surtout pour les éléments allant de La à $\mathrm{Eu})$.

2. une pureté insuffisante des $R E$, qui se présentent parfois sous la forme de mélanges des différents RE.

Les $R E$ de $G d$ à Lu sont plus stables chimiquement (ce qui facilite leur manipulation) et les méthodes de purification sont plus efficaces que pour les précédentes.

a. Terres rares premiers éléments allant de La à Eu.

Ces RE appartiennent au même groupe cristallographique $\left(P 6_{3} / m m c\right)$ sauf l'europium (Fm3m). Si nous représentons les plus courtes distances entre les atomes RE de toute la série du groupe $P 6_{3} / \mathrm{mmc}$ en fonction des numéraux atomiques (établies expérimentalement) (Fig.1) [4] nous voyons que la courbe $d_{R E-R E}=\mathrm{f}(N)$, présente deux branches qui répondent aux équations suivantes :

$d_{\text {La-Gd }}=1.32238-0.0298946 \cdot \mathrm{N}+2.31471 \cdot 10^{-4} \cdot \mathrm{N}^{2}$
$\mathrm{~d}_{\text {Gd-Lu }}=1.56745-0.0342554 \cdot \mathrm{N}+2 \cdot 397 \cdot 10^{-4} \cdot \mathrm{N}^{2}$

II n'y a qu' $\mathrm{Yb}(\mathrm{N}=70)$ qui présente une anomalie. La dispersion des données (surtout pour La, Eu et Lu) est liée, probablement, aux impuretés présentes dans les éléments. Une telle régularité se retrouve pour la masse volumique des RE en fonction du numéro atomique (Fig.2) sauf pour Eu et Yb.

Un autre un exemple concerne les entropies standard des RE (Fig.3). La relation entre les entropies $S_{298}^{0}$ en fonction du numéro atomique des RE montre également deux branches indépendantes. Elles sont établies à partir des références $[5,6]$ et de résultats calorimétriques expérimentaux obtenus pour les éléments ayant subis une distillation et présentant une haute pureté : $\mathrm{Pr}, \mathrm{Er}$ et $\mathrm{Tm}$ [7-9]. Si la branche qui concerne des 'métaux lourds' de Gd à Lu (y compris

This is an Open Access article distributed under the terms of the Creative Commons Attribution-Noncommercial License (http://creativecommons.org/licenses/by-nc/3.0/), which permits unrestricted use, distribution, and reproduction in any noncommercial medium, provided the original work is properly cited. 
Yb) est assez correctement définie, l'autre branche des 'métaux légers' de La à Eu présente des résultats très dispersés, ce qui tend à confirmer les problèmes mentionnés plus haut.

$S_{298}^{o}(\mathrm{La}-\mathrm{Gd})=-3902+130.122 \cdot \mathrm{N}-1.06379 \cdot \mathrm{N}^{2} \quad( \pm 2$

$\mathrm{J} / \mathrm{mol}$ ) K pour La-Pr et $\pm 4 \mathrm{~J} / \mathrm{mol} \mathrm{K}$ pour Nd-Eu)

$S_{298}^{o}(\mathrm{Gd}-\mathrm{Lu})=29065.2-1378.55 \cdot \mathrm{N}+21.7754 \cdot \mathrm{N}^{2}-$

$0.114294 \cdot \mathrm{N}^{3}( \pm 1 \mathrm{~J} / \mathrm{mol} \mathrm{K})$

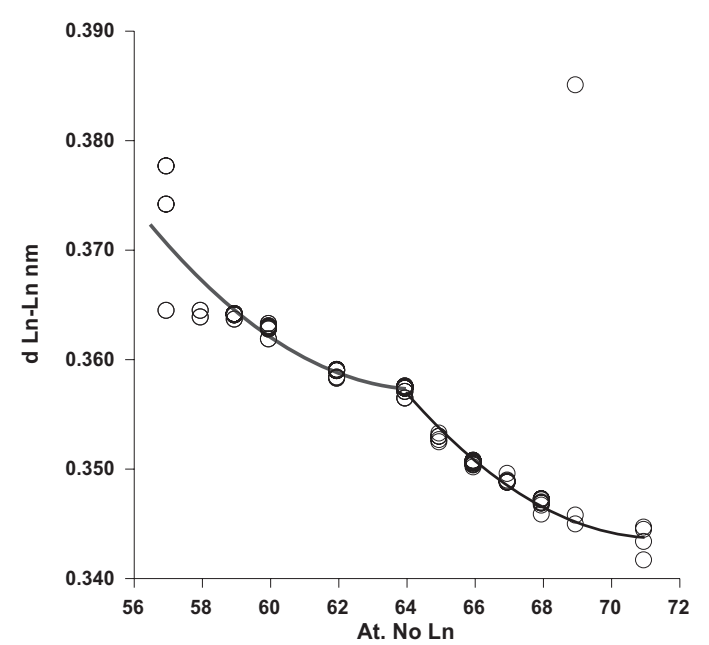

Fig.1. Plus courtes distances entre les atomes des RE purs en fonction de leurs numéros atomiques

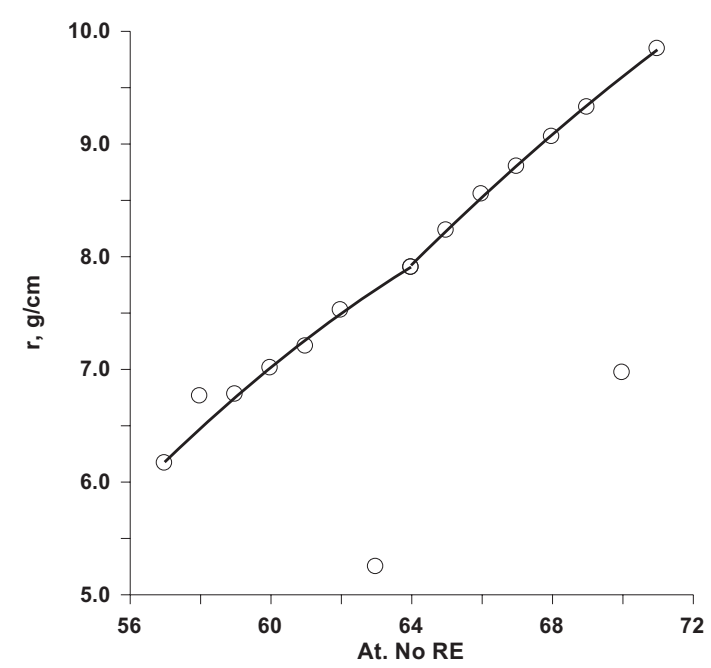

Fig.2. Masse volumique des RE en fonction des numéros atomique.

$\rho(N) L a-G d=-36.457+1.1935 \cdot N-7.8186 \cdot 10^{-3} \cdot N^{2}$ $\left(\mathrm{g} / \mathrm{cm}^{3}\right)(5)$

$\rho(N) G d-L u=-33.586+0.9866 \cdot N-5.2840 \cdot 10^{-3} \cdot N^{2}$

$\left(\mathrm{g} / \mathrm{cm}^{3}\right)(6)$

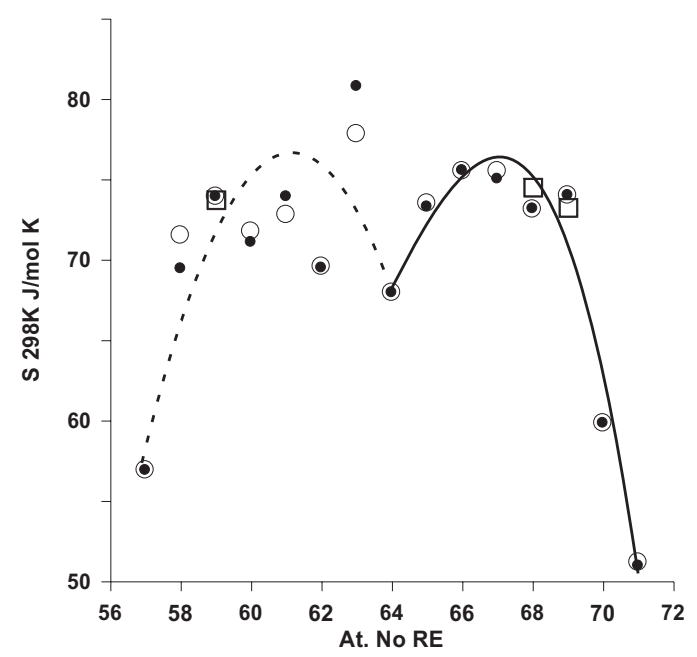

Fig. 3. Entropies $S_{298}^{0}$ des RE en fonction de leurs numéros atomiques $\circ-[5], \bullet-[6], \square-[7-9]$

\section{b. Alliages à base de terres rares.}

Les alliages des terres rares avec d'autres éléments du tableau périodique forment une grande série de phases isostructurales. Si nous prenons les alliages des systèmes RE-In nous pouvons noter qu'il existe 6 types de phases isostructurales en fonction de la composition Fig.4.

\section{c. Phases REln 3 .}

Prenons à titre d'exemple les phases $\mathrm{REIn}_{3}$. Elles présentent toutes une fusion congruente à l'exception de $\mathrm{Ybln}_{3}$ qui se décompose selon une réaction péritectique [4], les calculs sont basés sur $\operatorname{Re}_{.25} \ln _{.75}$.

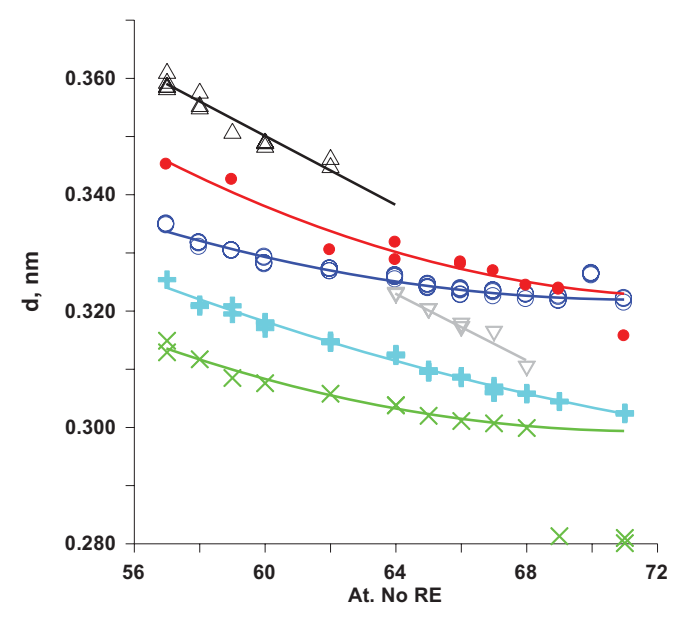

Fig.4. Plus courtes distances expérimentales entre des atomes des RE et In en fonction des numéraux atomiques dans les alliages $R E \ln _{3}$ [4] pour six types des phases des systèmes RE-In: $\bigcirc-1: 3$ (type $\mathrm{Cu}_{3} \mathrm{Au}$ ), $\times$ - 3:5 (type $\mathrm{Pu}_{3} \mathrm{Pd}_{5}$ ), - 1:1 (type CsCl), + - 2:1 (type $\mathrm{Ni}_{2} \mathrm{In}$ ), $\Delta$ - 3:1 (type $\left.\mathrm{Cu}_{3} \mathrm{Au}\right), \nabla-5: 3$ type $\mathrm{W}_{5} \mathrm{Si}_{3}$.

Les systèmes Eu-In et Yb-In n'entrent pas dans cette classification. Les phases Luln (type CuAu), $\mathrm{Tm}_{3} \mathrm{In}_{5}$ et $\mathrm{Lu}_{3} \mathrm{In}_{5}$ (type $\mathrm{Tm}_{3} \mathrm{Ga}_{5}$ ) ont leurs propres structures. 
Sur la figure 5 nous présentons les plus courtes distances entre atomes de RE et In [4], en fonction des numéros atomiques.

L'ensemble de ces valeurs peut être traité, soit par deux polynômes (7) et (8) soit par un seul (9), les valeurs marquées par un triangle ne sont pas retenues pour les calculs Nous observons une affinité 'génétique' des propriétés physico-chimiques entre les métaux RE purs et leurs alliages avec d'autres éléments.

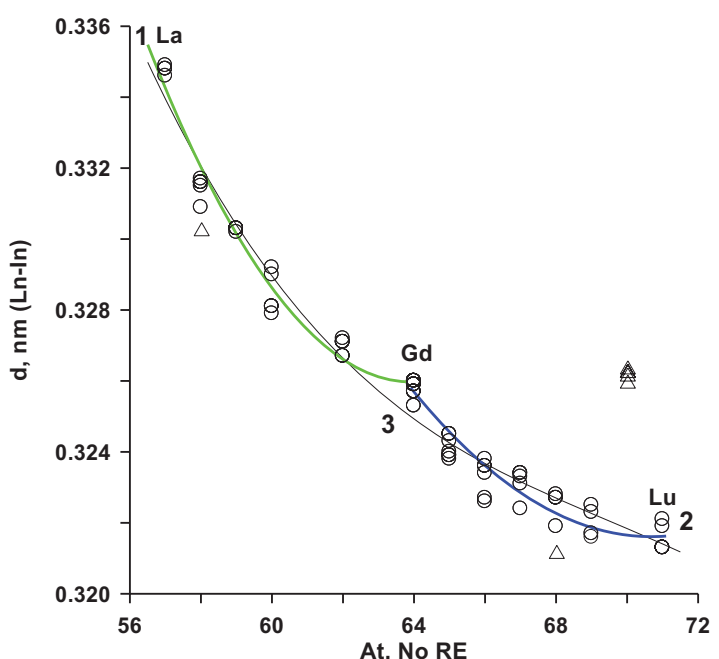

Fig.5. Plus courtes distances expérimentales entre atomes de RE et In en fonction des numéraux atomiques dans les alliages REIn ${ }_{3}$ [4].

$$
\begin{aligned}
& \text { Courbe } 1: d(\mathrm{~N}) \text { La-Gd }=1.03279-0.0221179 \cdot \mathrm{N}+ \\
& 1.73029 \cdot 10^{-4} \cdot \mathrm{N}^{2} \\
& \text { Courbe } 2: \mathrm{d}(\mathrm{N}) \mathrm{Gd}) \mathrm{Lu}=0.77749-0.012899 \cdot \mathrm{N}+ \\
& 9.1214 \cdot 10^{-5} \cdot \mathrm{N}^{2} \\
& \text { Courbe } 3: \mathrm{d}(\mathrm{N}) \text { La-Lu }=1.49878-0.049819 \cdot \mathrm{N}+ \\
& 7.13552 \cdot 10^{-4} \cdot \mathrm{N}^{2}-3.42453 \cdot 10^{-6} \cdot \mathrm{N}^{3}
\end{aligned}
$$

\section{d. Propriétés thermodynamiques des phases $\mathrm{REIn}_{3}$.}

Les propriétés thermodynamiques des alliages RE-In sont étudiées à l'aide des méthodes calorimétriques et potentiométriques, elles sont présentées dans le Tableau 1. Les enthalpies de formation de ces phases sont obtenues par deux méthodes : calorimétrie (à $298 \mathrm{~K}$ ) et potentiométrie (à $775 \mathrm{~K}$ ), nous pouvons donc déduire $\Delta C_{p}$ pour toutes les phases:

$\Delta C_{\mathrm{p}}(\mathrm{N})=\left(\Delta_{f} H_{775}^{o}(N)-\Delta_{f} H_{298}^{o}(N) /(775-298)\right.$

La température de $775 \mathrm{~K}$ est retenue comme valeur moyenne pour toutes les mesures potentiométriques entre 640-910K. Si ces mesures sont réalisées à une température supérieure à $910 \mathrm{~K}$, elles sont moins précises par suite des réactions d'échanges qui se produisent dans les piles [34]. Pour toutes les mesures, nous avons déterminé $(\Delta G(T, N), \Delta H(T, N), \Delta S(T, N)$, $\Delta C p\left(N, T=\right.$ const.) des phases $R E I_{3}$ sous forme d'un polynôme du troisième degré avec $\mathrm{T}$ - température en $\mathrm{K}$ et $\mathrm{N}$ - numéro atomiques de RE.
Les descriptions de ces fonctions avec une approximation linéaire [35] ou avec un polynôme du deuxième degré donnent des erreurs plus grandes que pour un polynôme du troisième degré.

Les équations (10-16) donnent les valeurs thermodynamiques calculées (Tableau 1). $\Delta_{f} G_{298}=-51.33-15.70 n+70.37 n^{2}-36.48 n^{3}$ $\Delta_{f} G_{775}=-45.42+0.97 n+10.66 n^{2}+4.05 n^{3}$ $\Delta_{\mathrm{f}} H_{298}=-54.98-19.47 n+97.46 n^{2}-58.12 n^{3}$ $\Delta_{f} H_{775}=-55.10-38.69 n+127.02 n^{2}-68.78 n^{3}$ $\Delta_{f} S_{298}=-12.25-12.67 n+90.92 n^{2}-72.61 n^{3}$ $\Delta_{f} S_{775}=-12.49-51.18 n+150.14 n^{2}-93.98 n^{3}$ $\Delta C_{p}=-0.25-40.30 n+61.96 n^{2}-22.35 n^{3}$

Nous avons utilisé un facteur normé $n=(N-57) / 14$

Les fonctions (calculées) $\Delta_{\mathrm{f}} G=f(T, N), \Delta_{\mathrm{f}} H=\mathrm{f}(\mathrm{T}, \mathrm{N})$, $\Delta_{f} S=f(T, N)$ et $\Delta C_{p}=f(N, T=C$ te $)$ sont présentées sur les Fig. 6-9.

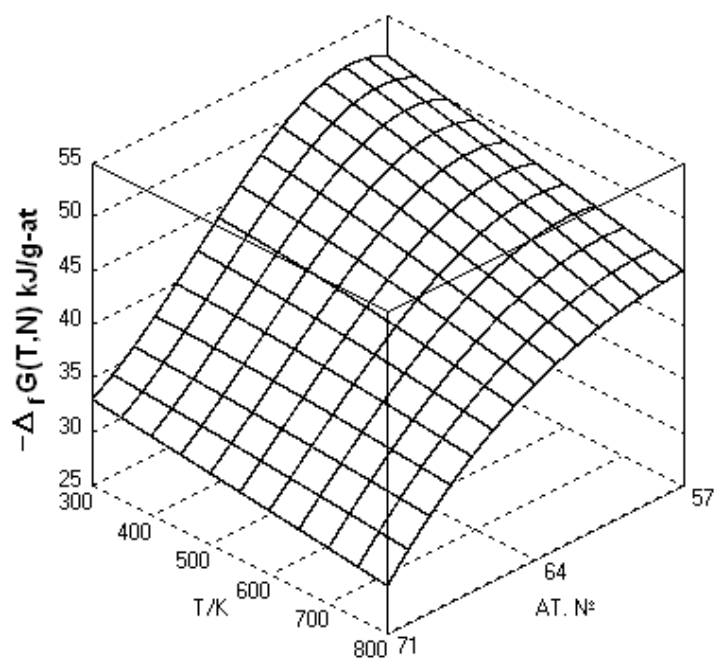

Fig.6. L'enthalpie libre $\Delta_{\mathrm{f}} G=f(T, N)$ RE $\ln _{3}$

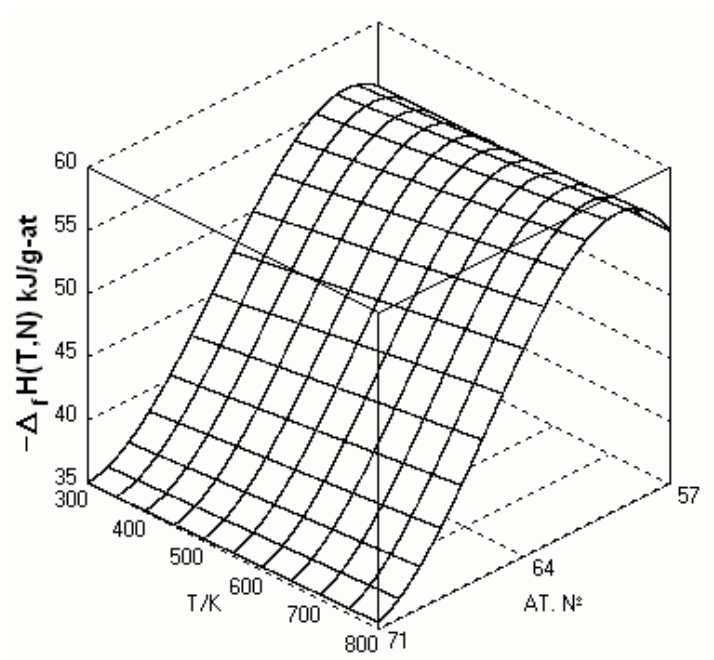

Fig.7. L'enthalpie $\Delta_{f} H=f(T, N)$ (calc) REln 3 
JEEP 2009

\begin{tabular}{|c|c|c|c|c|c|c|c|c|c|c|}
\hline \multicolumn{11}{|c|}{ Tableau.1 . Propriétés thermodynamiques des phases $\mathrm{Ln}_{.25} \mathrm{In}_{.75}$} \\
\hline Ln & At. № & $\begin{array}{l}-\Delta_{\mathrm{f}} H^{\circ} \text { exp. } \\
\mathrm{kJ} / \mathrm{mol}\end{array}$ & $\mathrm{T}, \mathrm{K}$ & $\begin{array}{c}-\Delta_{\mathrm{f}} G^{\circ}{ }_{775} \\
\exp \\
\mathrm{kJ} / \mathrm{mol}\end{array}$ & $\begin{array}{c}-\Delta_{\mathrm{f}} S^{\circ}{ }_{775} \\
\exp \\
\mathrm{J} / \mathrm{mol} \mathrm{K}\end{array}$ & $\begin{array}{l}\text { Reference, } \\
\text { méthode }\end{array}$ & $\begin{array}{c}-\Delta_{f} H^{\circ} \mathrm{T} \\
\text { calc. } \\
\mathrm{kJ} / \mathrm{mol}\end{array}$ & $\begin{array}{c}-\Delta_{\mathrm{f}} G^{\circ} \mathrm{T} \\
\text { calc. } \\
\mathrm{kJ} / \mathrm{mol}\end{array}$ & $\begin{array}{c}-\Delta_{\mathrm{f}} S^{\circ} \mathrm{T} \\
\text { calc. } \\
\mathrm{J} / \mathrm{mol} \mathrm{K}\end{array}$ & $\begin{array}{l}S^{\circ}{ }_{298} \\
\text { calc } \\
\mathrm{J} / \mathrm{mol} \mathrm{K}\end{array}$ \\
\hline \multirow{7}{*}{ La } & \multirow{7}{*}{57} & $52.2 \pm 3$ & 298 & - & - & [10] calor. & \multirow{7}{*}{$\begin{array}{l}55.0 \pm 3^{\prime} \\
55.1 \pm 3^{\prime \prime}\end{array}$} & \multirow{7}{*}{$\begin{array}{l}51.3 \pm 2^{\prime} \\
45.4 \pm 1^{\prime \prime}\end{array}$} & \multirow{7}{*}{$\begin{array}{l}12.3 \pm 3^{\prime} \\
12.5 \pm 2^{\prime \prime}\end{array}$} & \multirow{7}{*}{$\begin{array}{c}45.6 \\
48.9[5]\end{array}$} \\
\hline & & $58.6 \pm 4$ & 300 & - & - & [11] calor. & & & & \\
\hline & & $69.9 \pm 3$ & 298 & - & - & [14] calor. & & & & \\
\hline & & $53.7 \pm 3$ & $725-975$ & $45.16 \pm 1$ & $11.0 \pm 5$ & [15] EMF & & & & \\
\hline & & $53.7 \pm 3$ & 298 & - & - & [12] calor. & & & & \\
\hline & & 57.1 & 298 & 52.66 & 14.9 & [13] optim. & & & & \\
\hline & & 57.1 & 775 & 45.56 & 14.9 & [13] optim. & & & & \\
\hline \multirow{2}{*}{$\mathrm{Ce}$} & \multirow{2}{*}{58} & $50.1 \pm 3$ & 298 & - & - & [10] calor. & \multirow{2}{*}{$\begin{array}{l}55.9 \pm 3^{\prime} \\
57.2 \pm 4^{\prime \prime}\end{array}$} & \multirow{2}{*}{$\begin{array}{l}52.1 \pm 2^{\prime} \\
45.3 \pm 1^{\prime \prime}\end{array}$} & \multirow{2}{*}{$\begin{array}{l}12.7 \pm 3^{\prime} \\
15.4 \pm 4^{\prime \prime}\end{array}$} & \multirow{2}{*}{$\begin{array}{c}47.2 \\
51.8[5]\end{array}$} \\
\hline & & $60.3 \pm 3$ & $725-975$ & $45.29 \pm 1$ & $22 \pm 6$ & [16] EMF & & & & \\
\hline \multirow{4}{*}{$\operatorname{Pr}$} & \multirow{4}{*}{59} & $55.8 \pm 3$ & 298 & - & - & [10] calor. & \multirow{4}{*}{$\begin{array}{l}55.9 \pm 3^{\prime} \\
58.2 \pm 2^{\prime \prime}\end{array}$} & \multirow{4}{*}{$\begin{array}{l}52.2 \pm 2^{\prime} \\
45.1 \pm 1^{\prime \prime}\end{array}$} & \multirow{4}{*}{$\begin{array}{c}12.4 \pm 3^{\prime} \\
17.0 \pm 2^{\prime \prime}\end{array}$} & \multirow{4}{*}{48.9} \\
\hline & & $65.3 \pm 3$ & 298 & - & - & [19] calor. & & & & \\
\hline & & $57.9 \pm 1$ & $725-975$ & $45.28 \pm 1$ & $16.3 \pm 3$ & [17] EMF & & & & \\
\hline & & $58.2 \pm 1$ & $648-973$ & $45.26 \pm 1$ & $16.1 \pm 3$ & [29] EMF & & & & \\
\hline \multirow{3}{*}{$\mathrm{Nd}$} & \multirow{3}{*}{60} & $54.5 \pm 3$ & 298 & - & - & [10] calor. & \multirow{3}{*}{$\begin{array}{l}55.3 \pm 3^{\prime} \\
58.2 \pm 2^{\prime \prime}\end{array}$} & \multirow{3}{*}{$\begin{array}{l}51.8 \pm 2^{\prime} \\
44.7 \pm 1^{\prime \prime}\end{array}$} & & \\
\hline & & $58.5 \pm 2$ & $725-975$ & $44.77 \pm 1$ & $17.6 \pm 3$ & [18] EMF & & & $\begin{array}{l}11.5 \pm 3^{\prime} \\
17.5+2^{\prime \prime}\end{array}$ & 50.6 \\
\hline & & $58.5 \pm 2$ & $678-973$ & $44.7 \pm 1$ & $14.7 \pm 3$ & [21] EMF & & & & \\
\hline $\mathrm{Pm}$ & 61 & - & - & - & - & - & $\begin{array}{c}53.9 \pm 3^{\prime} \\
57.4 \pm 3^{\prime \prime}\end{array}$ & $\begin{array}{l}50.9 \pm 2{ }^{\prime} \\
44.2 \pm 1^{\prime \prime}\end{array}$ & $\begin{array}{l}10.1 \pm 3^{\prime} \\
17.0 \pm 3^{\prime \prime}\end{array}$ & 52.4 \\
\hline Sm & 62 & $49.8 \pm 3$ & 298 & - & - & [10] calor. & $\begin{array}{l}52.1 \pm 3^{\prime} \\
55.8 \pm 3^{\prime \prime}\end{array}$ & $\begin{array}{c}49.6 \pm 22^{\prime} \\
43.5 \pm 1^{\prime \prime}\end{array}$ & $\begin{array}{c}8.5 \pm 3^{\prime} \\
15.9 \pm 3^{\prime \prime}\end{array}$ & 53.8 \\
\hline & & $50.63 \pm 1.2$ & $640-823$ & $41.5 \pm 0.5$ & $11.8 \pm 1$ & [22] EMF & & & & \\
\hline $\mathrm{Gd}$ & 64 & $45.7 \pm 3$ & 298 & - & & [10] calor. & $\begin{array}{l}47.6 \pm 2^{\prime} \\
513+2^{\prime \prime}\end{array}$ & $\begin{array}{c}46.1 \pm 1^{\prime} \\
418+0.5^{\prime \prime}\end{array}$ & $\begin{array}{r}4.9 \pm 3^{\prime} \\
123+2^{\prime \prime}\end{array}$ & 55.5 \\
\hline & & $46.93 \pm 2$ & $673-823$ & $41.40 \pm 1$ & $7.2 \pm 5$ & [27] EMF & & & & \\
\hline & & $49.93 \pm 0.5$ & $643-793$ & $41.17 \pm 0.5$ & $10.4 \pm 3$ & [22] EMF & & & & \\
\hline $\mathrm{Tb}$ & 65 & $44.6 \pm 2.5$ & 298 & - & - & [10] calor. & $\begin{array}{l}45.1 \pm 22^{\prime} \\
48.6+2^{\prime \prime}\end{array}$ & $\begin{array}{l}44.1 \pm 1^{\prime} \\
406+1^{\prime \prime}\end{array}$ & $\begin{array}{l}3.4 \pm 3^{\prime} \\
102+2^{\prime \prime}\end{array}$ & 58.0 \\
\hline & & $48.5 \pm 1$ & $673-873$ & $41.36 \pm 0.5$ & $9.3 \pm 3$ & [28] EMF & & & & \\
\hline & & $44.8 \pm 3$ & 298 & - & - & [10] calor. & $42.7 \pm 3^{\prime}$ & $42.0 \pm 1^{\prime}$ & $2.1 \pm 4^{\prime}$ & \\
\hline Dy & 66 & $44.2 \pm 3$ & $640-900$ & $35.8 \pm 1$ & $10.8 \pm 5$ & [30] EMF & $45.8 \pm 2^{\prime \prime}$ & $39.3 \pm 1^{\prime \prime}$ & $8.3 \pm 3^{\prime \prime}$ & 60.0 \\
\hline$H$ & 67 & $40.6 \pm 2.5$ & 298 & - & - & [10] calor. & $40.3 \pm 2^{\prime}$ & $39.9 \pm 1^{\prime}$ & $1.4 \pm 4^{\prime}$ & 010 \\
\hline HO & 01 & $45.9 \pm 1.5$ & $670-950$ & $37.33 \pm 1$ & $11.0 \pm 5$ & [31] EMF & & $37.8 \pm 1 "$ & $6.7 \pm 4^{\prime \prime}$ & 61.0 \\
\hline & & $41.17 \pm 1.1$ & 644-804 & $36.25 \pm 0.5$ & $6.4 \pm 2$ & [24] EMF & & & & \\
\hline & & $38.9 \pm 2.5$ & 298 & - & & [10] calor. & & & & \\
\hline $\mathrm{Er}$ & 68 & $39.83 \pm 2.5$ & $673-873$ & $36.4 \pm 0.5$ & $4.4 \pm 3$ & [27] EMF & $38.3 \pm 22^{\prime}$ & $\begin{array}{c}37.9 \pm 1^{\prime} \\
361+y^{\prime \prime}\end{array}$ & $1.3 \pm 2^{\prime}$ & 60.8 \\
\hline & & $41.23 \pm 2.5$ & $673-755$ & $36.1 \pm 0.5$ & $6.6 \pm 2$ & [32] EMF & & & & \\
\hline & & $39.0 \pm 1$ & $675-925$ & $36.1 \pm 0.5$ & $4.5 \pm 3$ & [33] EMF & & & & \\
\hline $\mathrm{Tm}$ & 69 & $36.5 \pm 2$ & 298 & - & - & [10] calor. & $\begin{array}{l}36.7 \pm 2^{\prime} \\
38.3 \pm 3^{\prime \prime}\end{array}$ & $\begin{array}{l}36.1 \pm 1^{\prime} \\
34.2 \pm 1^{\prime \prime}\end{array}$ & $\begin{array}{c}2.0 \pm 2^{\prime} \\
5.2 \pm 2^{\prime \prime}\end{array}$ & 59.1 \\
\hline $\mathrm{Yb}$ & 70 & $39.7 \pm 2.5$ & 298 & - & - & [10] calor. & - & - & - & \\
\hline & & $36.37 \pm 0.5$ & $643-810$ & $31.05 \pm 0.5$ & $6.9 \pm 2$ & {$[25,26]$ EMF } & & & & \\
\hline & & $34.7 \pm 2$ & 298 & - & - & [10] calor. & $35.1 \pm 2^{\prime}$ & $33.1 \pm 1^{\prime}$ & $6.6 \pm 2^{\prime}$ & \\
\hline Lu & 71 & $35.3 \pm 1$ & $675-925$ & $28.9 \pm 1$ & $8.3 \pm 3$ & [33] EMF & $35.6 \pm 1^{\prime \prime}$ & $29.7 \pm 1^{\prime \prime}$ & $7.5 \pm 2^{\prime \prime}$ & 49.4 \\
\hline & & $31.4 \pm 2$ & $674-873$ & $28.6 \pm 2$ & $3.2 \pm 4$ & [29] EMF & & & & \\
\hline
\end{tabular}

Remarque : Les valeurs expérimentales avec les chiffres gras ont un poids compris entre 0,9 et 1 , non gras entre 0.3 et 0.8 , en italique -moins 0.3. Valeurs calculées : (') à $298 \mathrm{~K}$, (") à $775 \mathrm{~K}$.

On a utilisé pour le calcul des $S^{\circ}{ }_{298}\left(\mathrm{La}_{.25} \ln .75\right)$ les équations (3) et (4) qui correspondent à $S^{\circ}{ }_{298}(\mathrm{RE})$, les valeurs $\Delta_{\mathrm{f}} S^{\circ}{ }_{298}$ $\left(\mathrm{Laln}_{3}\right)$ sont prises du Tableau1 et $S^{\circ}{ }_{298}(\mathrm{In})=57.82 \mathrm{~J} / \mathrm{mol} \mathrm{K}$ de [5].

La température de $775 \mathrm{~K}$ est retenue comme valeur moyenne pour toutes les mesures potentiométriques. 


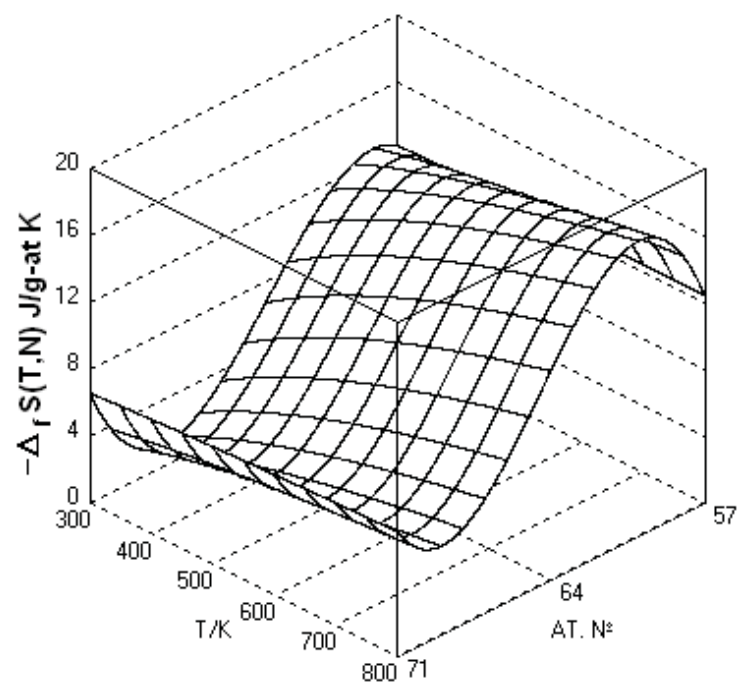

Fig.8. L'entropie $\Delta_{\mathrm{f}} S=\mathrm{f}(\mathrm{T}, \mathrm{N})$ REIn ${ }_{3}$

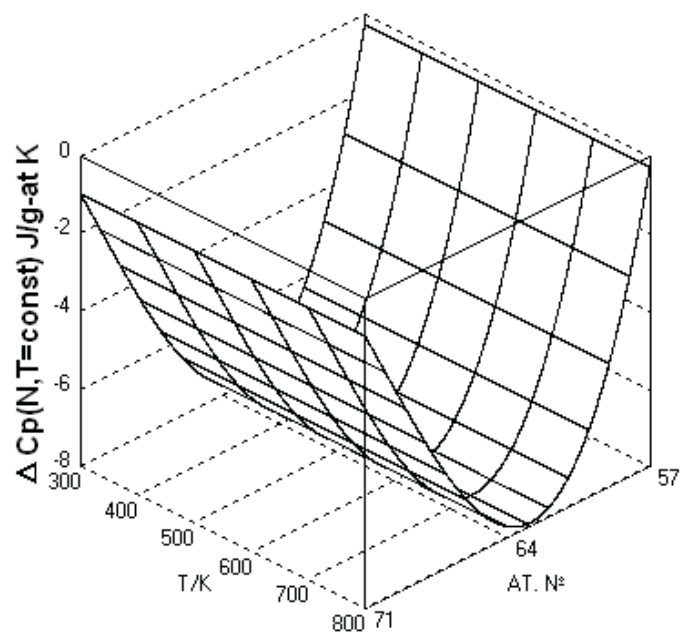

Fig.9. $\Delta C_{p}=f(N) R E \ln _{3}$

\section{Conclusion :}

1. A l'heure actuelle les méthodes de calculs thermodynamiques nécessitent de réaliser une optimisation de tout un ensemble de données pour chaque système afin de parvenir à une auto cohérence dans le cadre d'un model thermodynamique unique.

2. La méthode corrélative proposée qui est basée sur l'estimation des propriétés physico-chimique, convient pour toutes les phases isostructurales avec d'autres éléments.

3. Les valeurs thermodynamiques calculées sont recommandées pour les "handbooks " destinés aux métallurgistes.

4. Nous recommandons de remesurer $S_{298}^{0}$, avec des $\mathrm{RE}$ de haute pureté pour La, Ce, Nd, Sm. Les phases $R E I_{3}$ pour des métaux de RE légers s'oxydent au contact de l'air plus facilement que les métaux RE purs en donnant des oxydes de type $\mathrm{RE}_{2} \mathrm{O}_{3}$ et de l'indium libre. Les valeurs $S^{\circ}{ }_{298}$, citées dans [5] pour les phases
Laln $_{3}$ et Celn $n_{3}$ peuvent être erronées par le fait que les échantillons qui ont été utilisées lors des mesures calorimétriques pouvaient contenir des oxydes $\mathrm{La}_{2} \mathrm{O}_{3}$, $\mathrm{Ce}_{2} \mathrm{O}_{3} ; \quad\left(S_{298}^{o}\left(\mathrm{La}_{0.25} \mathrm{O}_{0.75}\right)=127.32 \mathrm{~J} / \mathrm{mol} \mathrm{K}\right)$.

\section{Références.}

[1] Vassiliev V.P. "Propriétés thermodynamique et équilibre entre phases des certains systèmes semiconducteurs et ceux métalliques" Thèse du docteur d'état. Moscou.2007. 350 P.

[2] Ansara I., Chatillon C., Lukas H.L., Nishizawa T., Ohtani H., Ishida K., Hillert M., Sundman B., Argent B.B., Watson A., Chart T.G. and Anderson T. A binary data base for III-V compounds semiconductor systems /I Calphad. 1994. V.18. №2. P.177-222.

[3] Li Ch., Li J.-B., Du Z., Zhang W. Thermodynamic assessment of the Ga-In-P system J. Phase Equilibria. 2000. V.21. No 4. P.357-363.

[4] Pauling File Binaries Edition. 2002.

[5] Gluchko V.P. Handbook "Thermic constancies of the substances” Edition VINITI. 1978.V.8.

[6] SGTE DATA for pur elements. CALPHAD 1991. V.15. P.317-430.

[7] Berezovski G.A., Burkhanov G.S., Kolchugina N.B., Paukov I.E., Tagaev A.B., Chistyakov O.D. Heat Capacity of praseodymium in a temperature range of 5.6-314 K. // Rus. J. Phys.Chem.1990. V. 64. No.10. P. 2636-2640.

[8] Berezovski G.A., Burkhanov G.S., Ilyasov S.Sh, Kolchugina N.B., Chistyakov O.D., Paukov I.E., Tagaev A.B. Heat Capacity of Thulium in a temperature range of 8-325 K // Rus. J. Phys.Chem.1991. V. 65. No.6. P. 1698-1703.

[9] Berezovski G.A. Boyarski L.A., Burhhanov G.S., Kazakov A.M., Kolchugina N.B., Paukov I.E., Chistyakov O.D. Heat Capacity of Erbium in a temperature range of $5-300 \mathrm{~K}$. // Rus. J. Phys.Chem.1993. V. 67. No.11. P.2153-2156.

[10] Palenzona A., Cirafici S. Dynamic differential calorimetry of intermetallic compounds // Thermochim. Acta. 1974. V.9. P. 419-425.

[11] Borsese A., Galabretta A., Delfino S., Ferro R. Measurements of heats of formation in the lantanium-indium system//J.Less-Common Metals.1977.V.51.P.45-49.

[12] Meschel S.V., Kleppa O.J. Standard enthalpies of formation of some metal indium compounds by direct synthesis calorimetry // J.Alloys Comp. 2002. V.333.P.91-98.

[13] Ying Wei, Xuping Su, Fucheng Yin, Zhi Li, Xianping Wu, Chuntao Chen. Thermodynamic assessment of the La-In system // J. Alloys Comp. 2002.V.333.P.118-121.

[14] Novozhenov V.A. Physicochemical Study of La-In and La-Ga alloys // Scientific and practical conference 
"Young scientists and experts of Tomsk region in the ninth five-years period». Theses of reports. Section chemistry. Tomsk. 1975. P.70-72.

[15] Degtjar V. A, Vnuchkova L. A., Bayanov A.P., Serebrennikov V.V. // Thermodynamics of systems La-In and La-TI // Izv.AN SSSR.Metals.971.No4.P.149-153.

[16] Degtjar V. A:, Vnuchkova L. A, Bayanov A.P., Serebrennikov V.V. Thermodynamic research of liquid Ce-In alloys // Rus. J. Phys.Chem.1971.V.45.No 6. P.1594.

[17] Degtjar V. A, Bayanov A.P., Vnuchkova L. A, Serebrennikov V.V. Thermodynamics of liquid Pr-In alloys // Rus. J. Phys.Chem.. 1971. T.45. No 7. P.1816-1818.

[18] Degtjar V.A, Bayanov A.P., Serebrennikov V.V. Thermodynamics Interaction of Neodymium with Indium // Works of Tomsk university.1971.V.204. P.401-402.

[19]Serebrennikov V., Novozhenov V.A, Shkolnikova T.M. Enthalpies of formation Pr-In alloys // Rus. J. Phys.Chem.1977.V.50.No9.P2401-2402.

[20] Kober V. I, Nichkov I. F, Raspopin S. P, Kuzminyh V.M. Thermodynamic properties of the saturated solutions of praseodymium with low-melting metals // Thermodynamics of the metal systems. AlmaAta. Publishing house "Science" 1979.P.67-71.

[21] Kober V. I, Nichkov I. F, Raspopin S. P, Kuzminyh V.M. Thermodynamic properties of the saturated solutions of praseodymium with low-melting metals // Thermodynamics of the metal systems. Alma-Ata. Publishing house "Science" 1979. P.72-76.

[22] Vassiliev V.P., Vu Dinh Khue. Thermodynamic properties of the system Gd-In in the region up to 50 at\% Gd // Izv.AN SSSR. Neorg.Material. 1985.V.21. No7. P.1144-1149.

[23] Vassiliev V.P., Khramstova L.A., Morozova V.V. Thermodynamic properties of intermetallic phases in the terbium-indium system in the range up to 50 atomic percentage of terbium // Vest.Mosk.Univ.Ser.2,Chim., 1986, v.27, (1), p.38-42.

[24] Vassiliev V.P., Vu Dinh Khue, Gerassimov Ja.I. Thermodynamic properties of the intermetallic Erln $\mathrm{n}_{2,5}$ and ErIn phases in the system erbium-indium. // Vest. Mosk. Univ. Ser.2. Chem. 1982. V.23.No 1.P.17-22.

[25] Vu Dinh Khue, Vassiliev V.P., Gerassimov Ja.I. Thermodynamic properties $\mathrm{Lu}_{2} \mathrm{In}_{5}$ and Luln. // Dokl.AN SSSR. 1982. V.269. No1. P.123-128.
[26] Vassiliev V.P., Vu Dinh Khue, Gerassimov Ja.I. A study of phase equilibrium and thermodynamic properties of alloys in the system lutecium-indium at the temperatures to 800K // J.Phis.Chem., 1985, V.59. No 11. P.2694-2699.

[27] Bayanov A.P., Afanasiev J.A, Pogorelaya N.M. Thermodynamic Study of the Gadolinium and Erbium compounds with Indium by EMF method // Rus. J. Phys. Chem. 1973. V.47. P. 2105-2107.

[28] Bayanov A.P., Ganchenko E. N, Afanasiev J.A. Thermodynamic Study of the Terbium alloys with Indium and Lead by EMF method // J. Phys. Chem. 1976. V.50. No 9. P.2381-2382.

[29] Bayanov A.P, Ganchenko E.H, Afanasiev J.A, Parhomenko T.A, Soboleva N.A. Study of the Thermodynamic properties Luln $_{3}$ by EMF method // Rus. J. Phys. Chem. 1975. T.49. Вып.2. S.202-203.

[30] Yamshchikov L.F., Lebedev V.A, Sattarov F. N, Kurochkin S.V. Thermodynamic properties of the liquid dysprosium alloys with low-melting elements // X AllUnion conference. "Problems of the calorimetry and chemical thermodynamics». Theses of reports V. 2. Chernogolovka. 1984. P. 521-523.

[31] Yamshchikov L.F., Lebedev V.A, Raspopin S.P., Raspopin S.P, Arhipov P.A Thermodynamic properties of liquid Holmium alloys with low-melting elements // V All-Union meeting on thermodynamics of metal alloys. Theses of reports. M. 1985. P.14-18.

[32] Yamshchikov L.F., Lebedev V.A, Nichkov I.F., Raspopin S.P., Karmanov Thermodynamics Properties of liquid erbium alloys with low-melting elements // Thermodynamics of metal systems. AlmaAta, Publishing house "Science" 1979. P.181-185.

[33] Vdovkina S.P. Thermodynamic properties of erbium - indium and lutecium - indium alloys. Materials of regional scientific and practical conference "Young scientists and experts to a national economy» Tomsk. 1977. V.1. P.4-6.

[34] Vassiliev V. Problèmes expérimentaux de la méthode potentiométrique avec l'électrolyte liquide lors de l'étude des alliages métalliques et semi-conducteurs // J. Phys. IV France 2004. V.113.P.91-95.

[35] Vassiliev V.P. On some particularities of phase diagrams and enthalpies of formation of intermediate phases in systems lanthanide-indium in the region of 0 50 at\% of Ln // Vest. Mosk. Univ. Ser.2.Chem. 1989. V.30. No 2. P.115-121. 